\title{
Somatic Mosaicism in the Pathogenesis of de novo Cerebral Arteriovenous Malformations: A Paradigm Shift Implicating the RAS-MAPK Signaling Cascade
}

\author{
Keyan Peterson Stephanie Coffman Aqib Zehri Anthony Anzalone \\ Zhidan Xiang Stacey Wolfe
}

Department of Neurosurgery, Wake Forest School of Medicine, Winston-Salem, NC, USA

\section{Keywords \\ Arteriovenous malformations · Angiogenesis · Vascular biology $\cdot$ Somatic $\cdot$ Mosaicism $\cdot$ De novo mutation}

\begin{abstract}
Cerebral arteriovenous malformations (AVMs) are leading causes of lesional hemorrhagic stroke in both the pediatric and young adult population, with sporadic AVMs accounting for the majority of cases. Recent evidence has identified somatic mosaicism in key proximal components of the RASMAPK signaling cascade within endothelial cells collected from human sporadic cerebral AVMs, with early preclinical models supporting a potential causal role for these mutations in the pathogenesis of these malformations. Germline mutations that predispose to deregulation of the RAS-MAPK signaling axis have also been identified in hereditary vascular malformation syndromes, highlighting the key role of this signaling axis in global AVM development. Herein, we review the most recent genomic and preclinical evidence implicating somatic mosaicism in the RAS-MAPK signaling pathway in the pathogenesis of sporadic cerebral AVMs. Also, we review evidence for RAS-MAPK dysregulation in hereditary vascular malformation syndromes and present a hy-
\end{abstract}

karger@karger.com www.karger.com/ced

Karger $\frac{1}{\%}$

GOPEN ACCESS
(C) 2021 The Author(s)

Published by S. Karger AG, Basel

This is an Open Access article licensed under the Creative Commons Attribution-NonCommercial-4.0 International License (CC BY-NC) (http://www.karger.com/Services/OpenAccessLicense), applicable to the online version of the article only. Usage and distribution for commercial purposes requires written permission. pothesis suggesting that this pathway is central for the development of both sporadic and syndrome-associated AVMs. Finally, we examine the clinical implications of these recent discoveries and highlight potential therapeutic targets within this signaling pathway.

(c) 2021 The Author(s)

Published by S. Karger AG, Basel

\section{Introduction}

Cerebral arteriovenous malformations (AVMs) are high-flow vascular lesions characterized by direct connections between arteries and veins with an absence of intervening capillary networks. AVMs remain a leading cause of lesional hemorrhagic stroke in the pediatric and young adult populations, without effective medical therapies. Understanding the molecular basis of these lesions is a key step in the development of novel targeted medical interventions. The genetic basis of cerebral AVM development remains unknown. Current knowledge suggests that significant interplay between genetics and environment ultimately contributes to mature lesion formation. AVMs that develop as part of familial vascular malforma-

Keyan Peterson

Department of Neurosurgery, Wake Forest School of Medicine

1 Medical Center Blvd.

Winston-Salem, NC 27157 (USA)

kapeters@wakehealth.edu 
tion syndromes are caused by known germline, or inherited, mutations; however, these lesions represent a low percentage of total AVM cases. The majority of cerebral AVMs are sporadic, that is, they arise in patients without a known family history of vascular disease. Until recently, potentially causal mutations had not been identified in cells from human sporadic cerebral AVMs. The discovery and interrogation of the somatic mosaicism present in sporadic cerebral AVMs has become an area of increasing research interest.

Previous studies have suggested that AVMs, once thought to be congenital anomalies, may form de novo or even recur after resection. Endothelial tissue collected from patient AVMs has demonstrated significant potential for proliferation and neoangiogenesis, suggesting that these lesions are dynamic in nature and responsive to the local environment [1-3]. While the genetic underpinnings of sporadic cerebral AVM formation are still being elucidated, our understanding of the molecular basis of these lesions is evolving by leveraging advanced genome sequencing techniques to detect low-fraction allelic variants present in tissue from human AVMs. Signaling abnormalities in transforming growth factor beta 1 (TGF $\beta$ 1)/bone morphogenetic protein [4], vascular endothelial growth factor (VEGF) $[1,2,4,5]$, platelet-derived growth factor [6], Notch [7], Eph/Ephrins [8], and the Sonic Hedgehog family proteins [9] have all been linked in varying degrees to AVM pathogenesis. Downstream alterations in transcription factors like the SMAD family proteins [10] and the phosphatidylinositol 3-kinase-protein kinase b-mammalian target of rapamycin (PI3KAKT-mTOR) signaling cascade [11] are also associated with aberrant vascular patterning. The intertwined relationships between various genes, proteins, and their downstream signaling components underscore the complexity and heterogeneity inherent in the study of AVM pathogenesis.

The RAS-RAF-MEK-ERK (RAS-MAPK) signaling sequence is integral in development and cell proliferation. While acquired over-activating mutations in the RASMAPK signaling axis are known to promote unrestricted growth in tumors [12], inherited or germline RAS-MAPK pathway-activating mutations have already been implicated in developmental vascular malformation syndromes such as capillary malformation-AVM (CMAVM) syndrome $[13,14]$. Recently, a high prevalence of somatic RAS-MAPK-activating mutations have been identified in sporadic cerebral and extracranial AVMs, providing a potential signaling link between sporadic and syndrome-associated cases of AVM formation [15-22].
In this review, we discuss the role of RAS activity in vascular development, with a focus on emerging evidence implicating key proximal components of the RAS-MAPK signaling cascade in the pathogenesis of sporadic AVMs. Also, we review evidence for RAS-MAPK dysregulation in hereditary vascular malformation syndromes and present a hypothesis suggesting that this pathway is central for the development of many sporadic and syndromeassociated AVMs. Finally, we examine the clinical implications of these recent discoveries and highlight potential therapeutic targets within this signaling axis.

\section{RAS Signaling and Vascular Development}

The RAS proteins (KRAS, HRAS, and NRAS) are monomeric GTPases that interact with receptor tyrosine kinases and are primarily controlled by growth factors such as VEGF, fibroblast growth factor, and platelet-derived growth factor. Once active, the RAS proteins act through multiple downstream effector pathways, including the RAS-MAPK and PI3K-AKT-mTOR signaling cascades, to produce changes in cellular proliferation, differentiation, and angiogenesis [23-25]. RAS is responsible for stimulating a pro-angiogenic phenotype in endothelial cells, and VEGF, a crucial mediator of angiogenesis, relies on RAS activation for proper signal transduction [25]. Previous investigations have demonstrated that a careful balance between RAS-MAPK and PI3K-AKT-mTOR signaling underlies arterial and venous vascular specification [26] and that downstream of RAS, MAPK-ERK activity is a potent regulator of VEGF expression and angiogenesis [27].

RAS proteins have a close signaling relationship with the EphrinB2-EphB4-RASA1 signaling axis, which is rapidly emerging as one of the most important pathways in arteriovenous specification and vasculogenesis $[28,29]$. The development of the cerebrovascular tree appears to rely critically on the interaction between the ligand EphrinB2 and the receptor EphB4 [8]. Among other cellular functions, the EphrinB2-EphB4 interaction allows intracellular binding of EphB4 to its direct downstream effector, RASA1, a GTPase-activating protein that functions to inactivate RAS by enhancing intrinsic GTPase activity [30]. The cumulative effect of RAS stimulation via growth factors and the negative regulation by RASA1 and EphB4 results in a net downstream signal that regulates gene transcription and, in concert with other signaling pathways, modulates angiogenesis (shown in Fig. 1). 


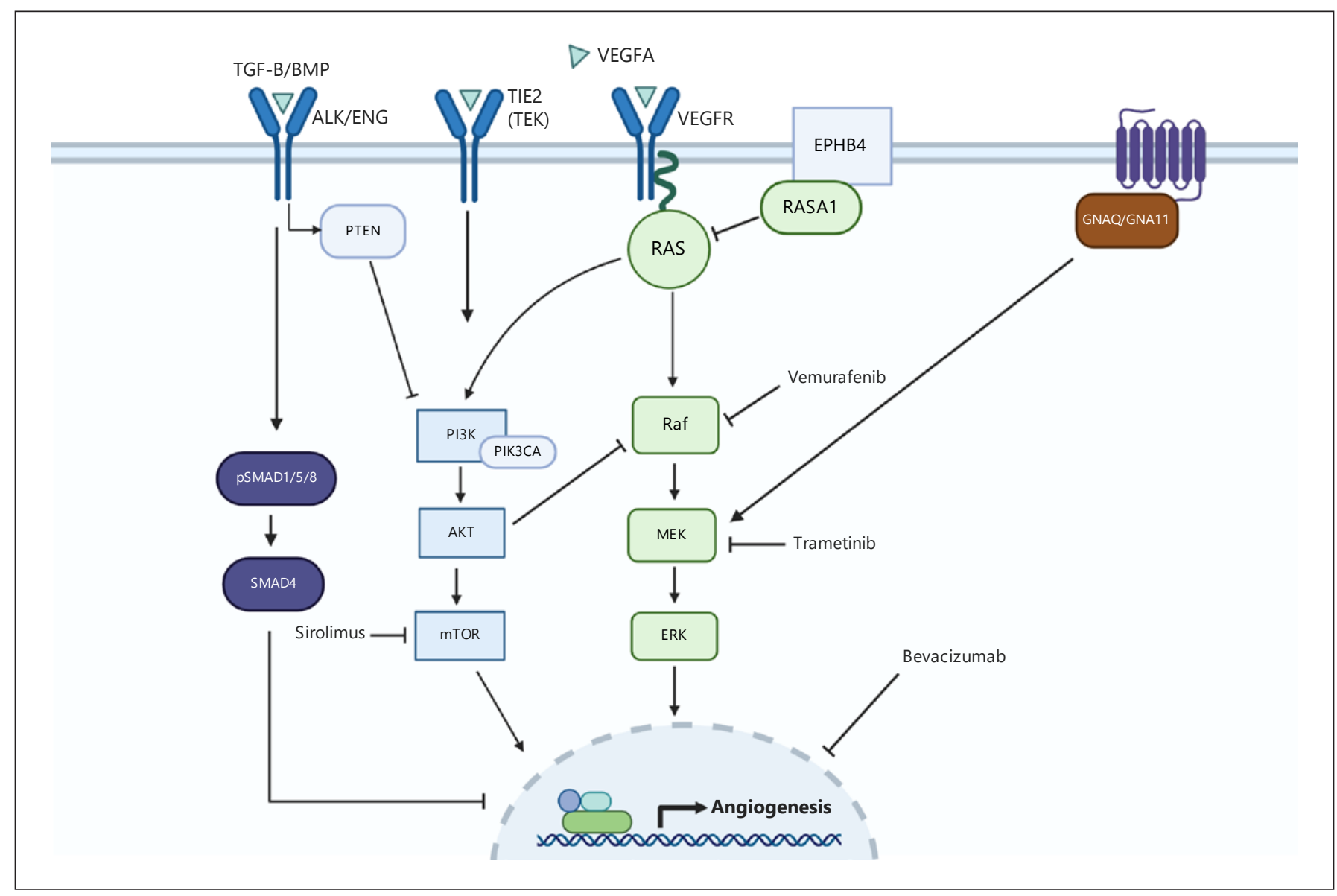

Fig. 1. Normal angiogenic signaling pathways and proposed therapeutic targets. VEGF signal transduction relies on RAS activation, which in turn activates the RAS-MAPK and PI3K-AKT-mTOR (PI3K) signaling cascades to promote angiogenesis. Gain-of-function mutations that activate RAS-MAPK, or loss-of-function mutations in EPHB4 and RASA1 (as in CM-AVM syndrome), result in upregulation of RAS-MAPK signaling, pERK formation, and the development of AVMs. Normally, TGF $\beta$ signaling opposes angiogenesis via canonical SMAD activity and via PTEN-mediated inhibition of PI3K signaling. Inactivating mutations in TGF $\beta$ family receptors ALK/ENG (as in HHT) result in a loss of the antiproliferative effects of SMAD. These mutations also result in recip-

\section{Somatic RAS-MAPK Mutations in Sporadic Cerebral AVMs}

The notion of a genetic mutation in a small subset of cells within a tissue leading to the development of pathology is well-established for many diseases, including cancer. These de novo, or post-zygotic, mutations are known to occur diffusely in human tissues and may occur during any stage of life, thus predisposing to lesion formation if not corrected by endogenous repair mechanisms. Until rocal increases in PI3K signaling and demonstrably increased pERK levels through a VEGF-dependent mechanism. The signaling commonalities between sporadic AVMs, CM-AVM, and HHT suggest a shared molecular basis underlying AVM development. Targeting the RAS-MAPK signaling cascade to inhibit pERK upregulation may represent a viable option to stabilize, obliterate, or reverse a significant number of AVMs. VEGF, vascular endothelial growth factor; TGF $\beta$, transforming growth factor beta; HHT, hereditary hemorrhagic telangiectasia; AVM, arteriovenous malformation; CM-AVM, capillary malformation-AVM; PI3K-AKTmTOR, phosphatidylinositol 3-kinase-protein kinase b-mammalian target of rapamycin; RAS-MAPK, RAS-RAF-MEK-ERK. recently, the study of AVM pathogenesis has been primarily geared towards examining germline, or whole-organism, mutations that are associated with the development of hereditary vascular malformation syndromes. This is despite the fact that the majority of AVMs arise sporadically, in patients without a family history of disease. Recent advances in next-generation sequencing technology have allowed for the identification of somatic mosaicism of RAS-MAPK signaling components within endothelial cells from patient sporadic cerebral AVMs. 
Table 1. Next-generation sequencing for RAS-MAPK variants in human sporadic AVMs

\begin{tabular}{|c|c|c|c|c|c|}
\hline \multirow[t]{2}{*}{ Reference } & \multicolumn{3}{|c|}{ RAS-MAPK variants identified } & \multirow[t]{2}{*}{ Tissue prep } & \multirow[t]{2}{*}{ Sequencing/coverage } \\
\hline & main cohort & $\begin{array}{l}\text { validation } \\
\text { cohort }\end{array}$ & total & & \\
\hline Al-olabi et al. [16] & $\begin{array}{l}9 / 25 ; \mathrm{i} K A S, \\
B R A F, M A P 2 K 1\end{array}$ & $\mathrm{~N} / \mathrm{A}$ & $9 / 25(36 \%)$ & $21 \mathrm{FFPE}, 4 \mathrm{FF}$, paired WB & $\begin{array}{l}\text { SureSeq solid tumor panel, Sanger for confirmation, } \\
500 \times \text { depth }\end{array}$ \\
\hline Couto et al. [19] & $\begin{array}{l}16 / 25 ; M A P 2 K 1 \\
(M E K 1)\end{array}$ & $\mathrm{N} / \mathrm{A}$ & $16 / 25(64 \%)$ & $\mathrm{FF}$, paired saliva and $\mathrm{WB}$ & WES, WGS, and ddPCR for confirmation \\
\hline Goss et al. [20] & $\begin{array}{l}10 / 16 ; K R A S \\
B R A F\end{array}$ & N/A & $10 / 16(62.5 \%)$ & $\mathrm{FF}$, paired WB & $\begin{array}{l}\text { MIP-Seq for } 5 \text { RAS-MAPK-encoding genes, ddPCR } \\
\text { for confirmation, and MAF }>1 \% \text { considered positive }\end{array}$ \\
\hline Hong et al. [17] & $\begin{array}{l}27 / 31 ; K R A S \\
B R A F\end{array}$ & N/A & $27 / 31(87.1 \%)$ & FF, paired WB & $\begin{array}{l}\text { Exome sequencing of } 422 \text {-gene solid tumor panel, } \\
\text { ddPCR for confirmation, and } 1,000 \times \text { depth }\end{array}$ \\
\hline Nikolaev et al. [15] & $29 / 39 ; K R A S$ & $\begin{array}{l}16 / 33 \\
K R A S\end{array}$ & $45 / 72(62.5 \%)$ & $\begin{array}{l}\text { Main cohort: FF and paired } \\
\text { WB, validation cohort: FFPE }\end{array}$ & WES and ddPCR for confirmation \\
\hline Oka et al. [22] & $38 / 61 ; K R A S$ & $\mathrm{~N} / \mathrm{A}$ & $25 / 61(41 \%)$ & FFPE & ddPCR for KRASG12V, KRASG12D \\
\hline Priemer et al. [21] & $6 / 21 ; K R A S$ & $\mathrm{~N} / \mathrm{A}$ & $6 / 21(28.6 \%)$ & FFPE & $\begin{array}{l}\text { Therascreen KRAS RGQ PCR kit to detect } 7 \text { specific } \\
\text { mutations in codons } 12 \text { and } 13 \text { of exon } 2 \text { of the } \\
\text { KRAS oncogene }\end{array}$ \\
\hline
\end{tabular}

FFPE, formalin-fixed paraffin-embedded; FF, fresh frozen; WB, whole blood; WES, whole exome sequencing; WGS, whole genome sequencing; ddPCR digital droplet PCR; MIP-Seq. molecular inversion probe-sequencing; MAF, mean allelic fraction; AVMs, arteriovenous malformations. ${ }^{a}$ Includes only highflow vascular malformations characteristic of AVMs.

The etiology of these mutations remains unclear, though they are theorized to develop post-zygotically during embryonic development, or as late-somatic mutations due to DNA replication errors or secondary to local cellular stress leading to irreparable DNA damage. The cells harboring the resultant mutations are posited to over-activate angiogenetic signaling pathways and play a central role in the development of abnormal vascular patterning.

\section{Human Genome Sequencing: High Prevalence of \\ RAS-MAPK-Activating Mutations in Human \\ Sporadic Cerebral AVMs}

Advances in next-generation sequencing technology now allow for the detection of post-zygotic or late-somatic mutations in a subset of cells or even a single cell. As applied to AVMs, sequencing has revealed identification of previously undescribed somatic mutations in the RASMAPK signaling cascade within the endothelial cells of patients with sporadic intracranial and extracranial AVMs (shown in Table 1).

Nikolaev et al. [15] used whole exome sequencing to identify somatic-activating KRAS mutations in 45 of 72 cerebral AVMs sampled and in none of the 21 paired blood samples. Endothelial cells collected from these patients' AVMs demonstrated increased MAPK and PI3K signaling activity, as well as strong staining for ERK1/2 phosphorylation, regardless of the presence of a RAS mutation [15], designating the importance of the MAPK common molecular pathway in the pathogenesis of these lesions. Moreover, these cells exhibited a strong pro-angiogenic phenotype, as evidenced by the upregulation of genes related to angiogenesis and arteriovenous specification such as Notch. They also demonstrated a robust correlation with native cells stimulated by VEGF, indicating that even in the absence of VEGF stimulation, the KRAS-mutant cells behaved like angiogenic endothelial cells [15]. With deeper genome sequencing depth $(1,000 \times)$, Hong et al. [17] identified somatic-activating KRAS or BRAF mutations in $87 \%$ of the 31 patients with non-inherited cerebral AVMs, and $100 \%$ of the patients with spinal AVMs using targeted panel sequencing of 422 common tumor-related genes. BRAF is a key downstream effector of KRAS, and RAF activation is the next sequential step in the RAS-MAPK signaling cascade. Goss et al. [18] discovered similar KRAS and BRAF mutations in endothelial cells of $63 \%$ of the intracranial AVMs using molecular inversion probe sequencing. All 3 of these studies used digital droplet PCR to confirm the presence of RAS-MAPK mutants and identify missed variants. Premier et al. [21] identified KRAS mutations 
in 6/21 formalin-fixed paraffin-embedded tissue blocks from intracranial AVMs. The lower mutation prevalence in this study may be attributable to the use of DNA extracted from formalin-fixed paraffin-embedded tissue and the use of an assay that is less sensitive to a mean allelic fraction lower than $1 \%$. The variation in mutant prevalence across these studies may be due to differences in sampling technique and a lack of standardized threshold for designating a sample as mutant, with some studies using a mean allelic fraction $>1 \%$ and some counting fractions as low at $0.03 \%$. Finally, several studies have identified KRAS, HRAS, NRAS, BRAF, and MAP2K1 mutations in extracranial AVMs $[16,19,20]$. In these studies, the fast-flow lesions, more characteristic of AVMs, were more likely to harbor RAS-MAPK pathway mutations than the low-flow lesions reminiscent of venous or lymphatic malformations. While somatic-activating mutations in venous or lymphatic malformations have been identified, these mutations primarily dysregulate PI3K-mTOR signaling [31].

\section{Preclinical Studies: Elucidating a Causal Role for}

RAS-MAPK Signaling in Sporadic Cerebral AVM

Development

With the use of genomic sequencing alone, it is unclear whether mutations of the RAS-MAPK signaling axis are sufficient to cause sporadic AVM development. However, a recent study in mice and embryonic zebrafish supports the notion that mutations of this pathway can drive the formation of AVMs in otherwise healthy tissue. The authors demonstrated that endothelial-specific gain-of-function $\mathrm{KRAS}^{\mathrm{G} 12 \mathrm{D} / \mathrm{G} 12 \mathrm{~V}}$ mutations alone were sufficient to induce cerebral AVM formation. These arteriovenous shunts were shown to be dependent on the upregulation of VEGF-dependent angiogenic signaling [32]. The lesions produced in these mice harbored remarkably similar pathology to those found in human cerebral AVM tissue, with fistulous arteriovenous connections and markers of increased angiogenic signaling. An earlier murine model showed that endothelial-specific knock-in HRAS ${ }^{\mathrm{V} 12}$ mutations produced dilated, proliferative, vascular malformations of the brain that were highly prone to hemorrhage [33]. The cerebral-endothelial cells retrieved from these mice demonstrated significant phenotypic remodeling, including altered endothelial cell sprouting patterns and reduced pericyte recruitment, which has recently been demonstrated to contribute to the destabilization of AVMs [34]. The vascular pattern of these malformations differed significantly from the pathology identified in KRAS-driven AVMs, and the relative contribution of HRAS and NRAS isoforms on cerebral AVM development requires further exploration.

\section{RAS-MAPK Dysregulation Associated with Hereditary Vascular Malformation Syndromes}

\section{CM-AVM Syndromes: Loss of Negative Feedback \\ Promotes RAS-MAPK Signaling}

While the vast majority of AVMs arise sporadically, syndromic AVMs may be caused by germline alterations of genes involved in angiogenesis, with or without additional post-zygotic mutations. The CM-AVM syndrome is one of the RASopathy syndromes, caused by underlying mutations in the RAS-MAPK pathway, which presents in the first year of life with capillary malformations of the brain, spine, and soft tissues, along with high-flow intracranial AVMs or arteriovenous fistulas [13]. CMAVM syndrome occurs through autosomal-dominant inheritance of a loss-of-function mutation, followed by a second "hit" or loss of heterozygosity [35], with impairment of key regulatory proteins of the EphrinB2-EphB4RASA1 signaling pathway which appear to play a vital role in cerebrovascular development and regulating RAS signaling [36]. CM-AVM1 is associated with a heterozygous pathogenic variant of RASA1 in $>50 \%$ of the patients, while CM-AVM2 was more recently identified and appears to be caused by mutations in EphB4, the direct upstream effector of RASA1 [14]. These loss-of-function mutations in RASA1 and EphB4 each release RAS from negative feedback, allowing for unchecked RAS signaling activity that presumably drives lesion development.

\section{Hereditary Hemorrhagic Telangiectasia: Interplay}

between TGF- $\beta$ and RAS-MAPK Signaling

The phenotypic distribution of the vascular malformations in CM-AVM syndrome shares similarities with those found in hereditary hemorrhagic telangiectasia (HHT) $[14,37,38]$. HHT is a rare cause of unstable, multifocal cerebral and spinal AVMs in young patients [39, 40]. Greater than $90 \%$ of the HHT syndromes appear to arise due to germline mutations in TGF $\beta$ family receptors - endoglin or activin receptor-like kinase 1 - resulting in decreased TGF $\beta$ signaling and disrupted downstream activity of the anti-angiogenic SMAD transcription factors, leading to a pro-angiogenic phenotype [41]. Loss-offunction mutations in endoglin and activin receptor-like kinase 1, as in HHT, also appear to be associated with reciprocal increases in PI3K-AKT-mTOR and MAPK sig- 
naling through a VEGF-dependent mechanism [42-44]. Given that overactive RAS is similarly associated with decreased TGF $\beta$ signaling activity [33], and that RAS is a known VEGF-dependent activator of both the PI3KAKT-mTOR and RAS-MAPK signaling pathways in endothelial cells [45], it seems plausible that the dysregulated RAS-MAPK signaling may contribute to the pathogenesis of AVMs in these patients. There appears to be substantial overlap with regard to RAS-MAPK signaling dysregulation across both sporadic and syndrome-associated AVM pathology, highlighting the likely significant role of this pathway in the pathogenesis of these lesions.

\section{Therapeutic Targets}

As evidence for RAS-MAPK dysregulation in the pathogenesis of cerebral AVMs continues to unfold, the next step to bridging the translational research gap is to identify critical steps in the signaling pathway most amenable to therapeutic targeting. Because the over-activation of various components of the RAS-MAPK signaling cascade can be found in up to $30 \%$ of the cancer types ( $40 \%$ for melanomas), trials of new therapies that target and alter this signaling axis are rapidly evolving. However, trials of therapies for RAS-MAPK-mutant AVMs are limited by a relative paucity of preclinical models that accurately recapitulate these lesions' genetic basis. Our group is currently developing 2 novel mouse models of KRAS-mutant AVMs, utilizing viral vector technology, to explore the causal relationship between cerebral-endothelial KRAS mutations and subsequent sporadic AVM development. As more effective preclinical models are developed, trials of therapies aimed at disrupting this pathway can be prioritized. One novel method to treat RAS-MAPK-mutant AVMs may be to explore the use of RAS-MAPK pathway small molecule inhibitors alone or with adjuvant radiosurgery to sensitize them to obliteration, in a manner similar to MAPK-mutant brain-metastatic melanoma [46].

Recent research has demonstrated that blocking downstream RAS-MAPK signal transduction through inhibition of BRAF or MEK can restore blood flow in separate zebrafish models of RAS-MAPK-mutant AVMs [16, 32]. There are also recent reports demonstrating the use of small molecule inhibitors to treat pediatric AVMs, with mixed results $[47,48]$. In 1 case, a patient with a MAP2K1mutant AVM was treated with sirolimus (rapamycin) to suppress mTOR signaling with no effect, whereas treatment with the MEK inhibitor trametinib was effective in significantly reducing lesion volume, indicating that the MAP2K1 mutation exerted its effect through a pathway separate from mTOR signaling [47]. Anti-VEGF therapy with bevacizumab has also been shown to decrease aberrant angiogenesis in an animal model of cerebral AVMs in which VEGF was overexpressed [49]. Given the close relationship between VEGF and downstream RASMAPK signaling, targeting this pathway may be another viable strategy to stabilize these lesions. Collectively, these data provide biologic plausibility for RAS-MAPK pathway dysregulation as both a cause of cerebral AVM development and as a key therapeutic target in reestablishing normal vascular patterning. An invigorated understanding of the molecular basis of AVM formation should lead to further trials of novel or repurposed therapies aimed at targeting this pathway.

\section{Conclusion}

Genomic sequencing of human AVM tissue as well as existing animal models support the dysregulation of the RAS-MAPK common molecular pathway as a critical driver in the pathogenesis of sporadic cerebral AVMs. The extended replication cycle of endothelial cells may mean that fewer somatic alterations are required for a significant effect in disrupting angiogenic signaling and that relatively few mutations may be required for the development of these lesions. The degree of contribution of each individual mutation, the timing of mutation occurrence, tissue or cell-type location, and the recruitment of other signaling pathways undoubtedly contribute to the phenotypic heterogeneity between AVMs. Further, local hemodynamic forces, inflammation, trauma, and epigenetic factors are all likely to play a crucial role in their pathogenesis. More effective preclinical models, incorporating RAS-MAPK pathway mutations that better recapitulate the genetic basis of the disease, are needed to study further the contribution of these mutations to the development of cerebral AVMs and to facilitate the development and testing of therapeutic targets aimed at prevention and treatment.

Given the wave of recent findings implicating various steps in the shared RAS-MAPK common molecular pathway in the pathogenesis of cerebral AVMs, there are almost assuredly unidentified mutations that produce complex interactions with this signaling axis and result in the development of AVMs. However, there remain a significant number of lesions without a well-characterized genetic basis. Advances in genomic sequencing will continue to elucidate these yet unidentified contributors, 
shedding more light on the pathogenesis of AVM formation and potentially providing additional targets in the development of treatment modalities.

\section{Statement of Ethics}

The authors have no ethical conflicts to disclose.

\section{Conflict of Interest Statement}

The authors have no conflicts of interest to disclose.

\section{Funding Sources}

There were no funding requirements for this manuscript.

\section{Author Contributions}

Keyan Peterson: conceptualization, research, drafting, figure/ table preparation, revising, and editing. Stephanie Coffman: research, drafting, revising, and editing. Aqib Zehri: revising and editing. Anthony Anzalone: revising and editing. Zhidan Xiang: conceptualization and revising. Stacey Wolfe: conceptualization, research, drafting, revising, and editing.

\section{References}

1 Sonstein WJ, Kader A, Michelsen WJ, Llena JF, Hirano A, Casper D. Expression of vascular endothelial growth factor in pediatric and adult cerebral arteriovenous malformations: an immunocytochemical study. J Neurosurg. 1996 Nov;85(5):838-45.

2 Sure U, Butz N, Schlegel J, Siegel AM, Wakat JP, Mennel HD, et al. Endothelial proliferation, neoangiogenesis, and potential de novo generation of cerebrovascular malformations. J Neurosurg. 2001 Jun 1;94(6):972-7.

3 Sandalcioglu IE, Asgari S, Wende D, van de Nes JA, Dumitru CA, Zhu Y, et al. Proliferation activity is significantly elevated in partially embolized cerebral arteriovenous malformations. Cerebrovasc Dis. 2010;30(4): 396-401.

4 Wang K, Zhao S, Liu B, Zhang Q, Li Y, Liu J, et al. Perturbations of BMP/TGF- $\beta$ and VEGF/VEGFR signalling pathways in nonsyndromic sporadic brain arteriovenous malformations (BAVM). J Med Genet. 2018; 55(10):675-84

5 Coultas L, Chawengsaksophak K, Rossant J. Endothelial cells and VEGF in vascular development. Nature. 2005 Dec 15;438(7070):93745.

6 Yildirim O, Bicer A, Ozkan A, Kurtkaya O, Cirakoglu B, Kilic T. Expression of plateletderived growth factor ligand and receptor in cerebral arteriovenous and cavernous malformations. J Clin Neurosci. 2010 Dec 1;17(12): 1557-62.

7 Carlson TR, Yan Y, Wu X, Lam MT, Tang GL, Beverly LJ, et al. Endothelial expression of constitutively active Notch4 elicits reversible arteriovenous malformations in adult mice. Proc Natl Acad Sci U S A. 2005 Jul 12;102(28): 9884-9.

8 Kuijper S, Turner CJ, Adams RH. Regulation of angiogenesis by Eph-ephrin interactions. Trends Cardiovasc Med. 2007 Jul 1;17(5): $145-51$.
9 Lawson ND, Vogel AM, Weinstein BM. Sonic hedgehog and vascular endothelial growth factor act upstream of the notch pathway during arterial endothelial differentiation. Dev Cell. 2002 Jul 1;3(1):127-36.

10 Crist AM, Lee AR, Patel NR, Westhoff DE, Meadows SM. Vascular deficiency of smad4 causes arteriovenous malformations: a mouse model of hereditary hemorrhagic telangiectasia. Angiogenesis. 2018;21(2):363-80.

11 Limaye N, Kangas J, Mendola A, Godfraind C, Schlögel MJ, Helaers R, et al. Somatic activating PIK3CA mutations cause venous malformation. Am J Hum Genet. 2015 Dec 3; 97(6):914-21.

12 Dhillon AS, Hagan S, Rath O, Kolch W. MAP kinase signalling pathways in cancer. Oncogene. 2007 May;26(22):3279-90.

13 Eerola I, Boon LM, Mulliken JB, Burrows PE, Dompmartin A, Watanabe S, et al. Capillary malformation-arteriovenous malformation, a new clinical and genetic disorder caused by RASA1 mutations. Am J Hum Genet. 2003 Dec 1;73(6):1240-9.

14 Amyere M, Revencu N, Helaers R, Pairet E, Baselga E, Cordisco M, et al. Germline lossof-function mutations in EPHB4 cause a second form of capillary malformation-arteriovenous malformation (CM-AVM2) deregulating RAS-MAPK signaling. Circulation. 2017 Sep 12;136(11):1037-48.

15 Nikolaev SI, Vetiska S, Bonilla X, Boudreau E, Jauhiainen S, Rezai Jahromi B, et al. Somatic activating KRAS mutations in arteriovenous malformations of the brain. N Engl J Med. 2018;378(3):250-61.

16 Al-Olabi L, Polubothu S, Dowsett K, Andrews KA, Stadnik P, Joseph AP, et al. Mosaic RAS/ MAPK variants cause sporadic vascular malformations which respond to targeted therapy. J Clin Invest. 2018 Nov 1;128(11):5185.

17 Hong T, Yan Y, Li J, Radovanovic I, Ma X, Shao YW, et al. High prevalence of KRAS/ BRAF somatic mutations in brain and spinal cord arteriovenous malformations. Brain J Neurol. 2019 Jan 1;142(1):23-34.
18 Goss JA, Huang AY, Smith E, Konczyk DJ, Smits PJ, Sudduth CL, et al. Somatic mutations in intracranial arteriovenous malformations. PLoS One. 2019;14(12):e0226852.

19 Couto JA, Huang AY, Konczyk DJ, Goss JA, Fishman SJ, Mulliken JB, et al. Somatic MAP2K1 mutations are associated with extracranial arteriovenous malformation. Am J Hum Genet. 2017 Mar 2;100(3):546-54.

20 Goss JA, Konczyk DJ, Smits PJ, Kozakewich HPW, Alomari AI, Al-Ibraheemi A, et al. Intramuscular fast-flow vascular anomaly contains somatic MAP2K1 and KRAS mutations. Angiogenesis. 2019 Nov 1;22(4):547-52.

21 Priemer DS, Vortmeyer AO, Zhang S, Chang HY, Curless KL, Cheng L. Activating KRAS mutations in arteriovenous malformations of the brain: frequency and clinicopathologic correlation. Hum Pathol. 2019 Jul;89:33-9.

22 Oka M, Kushamae M, Aoki T, Yamaguchi T, Kitazato K, Abekura Y, et al. KRAS G12D or G12V mutation in human brain arteriovenous malformations. World Neurosurg. 2019 Jun;126:e1365-73.

23 Song M, Finley SD. Mechanistic insight into activation of MAPK signaling by pro-angiogenic factors. BMC Syst Biol. 2018 Dec 27; 12(1):145.

24 Serban D, Leng J, Cheresh D. H-ras regulates angiogenesis and vascular permeability by activation of distinct downstream effectors. Circ Res. 2008 Jun 6;102(11):1350-8.

25 Kawasaki K, Watabe T, Sase H, Hirashima M, Koide $\mathrm{H}$, Morishita $\mathrm{Y}$, et al. Ras signaling directs endothelial specification of VEGFR2+ vascular progenitor cells. J Cell Biol. $2008 \mathrm{Apr}$ 7;181(1):131-41.

26 Hong CC, Peterson QP, Hong JY, Peterson RT. Artery/vein specification is governed by opposing phosphatidylinositol-3 kinase and MAP kinase/ERK signaling. Curr Biol. 2006 Jul 11;16(13):1366-72. 
27 Sohn SJ, Sarvis BK, Cado D, Winoto A. ERK5 MAPK regulates embryonic angiogenesis and acts as a hypoxia-sensitive repressor of vascular endothelial growth factor expression. J Biol Chem. 2002 Nov 8;277(45):43344-51.

28 Wang HU, Chen ZF, Anderson DJ. Molecular distinction and angiogenic interaction between embryonic arteries and veins revealed by ephrin-B2 and its receptor Eph-B4. Cell. 1998 May 29;93(5):741-53.

29 Fish JE, Wythe JD. The molecular regulation of arteriovenous specification and maintenance. Dev Dyn. 2015;244(3):391-409.

30 Kawasaki J, Aegerter S, Fevurly RD, Mammoto A, Mammoto T, Sahin M, et al. RASA1 functions in EPHB4 signaling pathway to suppress endothelial mTORC1 activity. J Clin Invest. 2014 Jun 2;124(6):2774-84.

31 Castel P, Carmona FJ, Grego-Bessa J, Berger MF, Viale A, Anderson KV, et al. Somatic PIK3CA mutations as a driver of sporadic venous malformations. Sci Transl Med. 2016 Mar 30;8(332):332ra42.

32 Fish JE, Flores-Suarez CP, Boudreau E, Herman AM, Gutierrez MC, Gustafson D, et al. Somatic gain of KRAS function in the endothelium is sufficient to cause vascular malformations that require MEK but not PI3K signaling. Circ Res [cited 2020 Jul 19];127(6): 727-43. https://www.ahajournals.org/doi/ 10.1161/CIRCRESAHA.119.316500.

33 Li QF, Decker-Rockefeller B, Bajaj A, Pumiglia K. Activation of ras in the vascular endothelium induces brain vascular malformations and hemorrhagic stroke. Cell Rep. 2018 Sep 11;24(11):2869-82.

34 Winkler EA, Birk H, Burkhardt JK, Chen X, Yue JK, Guo D, et al. Reductions in brain pericytes are associated with arteriovenous malformation vascular instability. J Neurosurg. 2018 Dec 1;129(6):1464-74.
35 Lapinski PE, Doosti A, Salato V, North P, Burrows PE, King PD. Somatic second hit mutation of RASA1 in vascular endothelial cells in capillary malformation-arteriovenous malformation. Eur J Med Genet. 2018 Jan; 61(1):11-6.

36 Chen D, Teng JM, North PE, Lapinski PE, King PD. RASA1-dependent cellular export of collagen IV controls blood and lymphatic vascular development. J Clin Invest. 2019 Jun 11;129(9):3545-61.

37 Wooderchak-Donahue WL, Akay G, Whitehead K, Briggs E, Stevenson DA, O'Fallon B, et al. Phenotype of CM-AVM2 caused by variants in EPHB4 : how much overlap with hereditary hemorrhagic telangiectasia (HHT)? Genet Med. 2019 Sep;21(9):2007-14.

38 Hernandez F, Huether R, Carter L, Johnston T, Thompson J, Gossage JR, et al. Mutations in RASA1 and GDF2 identified in patients with clinical features of hereditary hemorrhagic telangiectasia. Hum Genome Var. 2015 Nov 5;2(1):15040-6.

39 Guttmacher AE, Marchuk DA, White RI. Hereditary hemorrhagic telangiectasia. N Engl J Med. 1995 Oct 5;333(14):918-24.

40 McDonald J, Bayrak-Toydemir P, Pyeritz RE. Hereditary hemorrhagic telangiectasia: an overview of diagnosis, management, and pathogenesis. Genet Med. 2011 Jul;13(7): 607-16.

41 McAllister KA, Grogg KM, Johnson DW, Gallione CJ, Baldwin MA, Jackson CE, et al. Endoglin, a TGF-beta binding protein of endothelial cells, is the gene for hereditary haemorrhagic telangiectasia type 1 . Nat Genet. 1994 Dec;8(4):345-51.

42 Ola R, Dubrac A, Han J, Zhang F, Fang JS, Larrivée B, et al. PI3 kinase inhibition improves vascular malformations in mouse models of hereditary haemorrhagic telangiectasia. Nat Commun. 2016 29;7:13650.
43 Jin Y, Muhl L, Burmakin M, Wang Y, Duchez AC, Betsholtz C, et al. Endoglin prevents vascular malformation by regulating flow-induced cell migration and specification through VEGFR2 signalling. Nat Cell Biol. 2017 Jun;19(6):639-52.

44 Choi EJ, Walker EJ, Shen F, Oh SP, Arthur HM, Young WL, et al. Minimal homozygous endothelial deletion of eng with VEGF stimulation is sufficient to cause cerebrovascular dysplasia in the adult mouse. Cerebrovasc Dis. 2012;33(6):540-7.

45 Bajaj A, Zheng Q, Adam A, Vincent P, Pumiglia K. Activation of endothelial ras signaling bypasses senescence and causes abnormal vascular morphogenesis. Cancer Res. 2010 May 1;70(9):3803-12.

46 Patel BG, Ahmed KA, Johnstone PA, Yu HH, Etame $\mathrm{AB}$. Initial experience with combined BRAF and MEK inhibition with stereotactic radiosurgery for BRAF mutant melanoma brain metastases. Melanoma Res. 2016;26(4): 382-6.

47 Lekwuttikarn R, Lim YH, Admani S, Choate KA, Teng JMC. Genotype-guided medical treatment of an arteriovenous malformation in a child. JAMA Dermatol. 2019;155(2):2567.

48 Maynard K, LoPresti M, Iacobas I, Kan P, Lam S. Antiangiogenic agent as a novel treatment for pediatric intracranial arteriovenous malformations: case report. J Neurosurg Pediatr. 2019 Oct 4:1-7.

49 Walker EJ, Su H, Shen F, Degos V, Amend G, Jun K, et al. Bevacizumab attenuates VEGFinduced angiogenesis and vascular malformations in the adult mouse brain. Stroke. 2012 Jul;43(7):1925-30. 